\title{
INTERMEDIALITÄT REVISITED Neue Perspektiven auf die Medienkunst
}

\author{
von CHRISTIANE HEIBACH
}

Janna Houwen: Film and Video Intermediality. The Question of Medium Specificity in Contemporary Moving Images, New York, London (Bloomsbury) 2017

Claudia Benthien, Jordis Lau, Maraike M. Marxsen: The Literariness of Media Art, New York (Routledge) 2019

Irena Barbara Kalla, Patrycja Poniatowska, Dorota Michułka (Hg.): On the Fringes of Literature and Digital Media Culture. Perspectives from Eastern and Western Europe, Leiden, Boston (Brill/Rodopi) 2018

Die Beobachtung, dass die interaktive Medienkunst ein hybrides Phänomen ist, in dem sich unterschiedliche Medien und Zeichensysteme mischen, die Barriere zwischen Kunst(-präsentation) und Betrachter_in durchlässig ist und somit der traditionelle Kunstbegriff ganz generell herausgefordert wird, ist inzwischen fast trivial zu nennen. Schon in den ersten Publikationen zu diesem Thema Ende der 199oer Jahre werden diese Aspekte hervorgehoben. ${ }^{1}$ Einig sind sich deren Verfasser_innen zudem darin, dass die (interaktive) (Medienkunst) auf ästhetische Strategien der Avantgarden zurückgreift, die auch schon vor dem Siegeszug der digitalen Medien mit ähnlichen Grenzüberschreitungen experimentieren. Die Entstehung von (Medienkunst) als Genre, also als Kunstart mit bestimmten Merkmalen, wird jedoch meist auf die 196oer Jahre datiert, als Künstler wie John Cage und Nam June Paik mit interaktiven Installationen und Performances auftreten, in denen die elektronischen
Medien Radio, Fernsehen und die gerade entstehende Videotechnologie entscheidende Rollen übernehmen. Demgemäß werden insbesondere Performanz, Interaktivität, Intermedialität und Selbstreflexivität als Unterschiede zu den klassischen Kunstformen betont.

All diese Kennzeichen finden sich aber auch schon in den Experimenten der frühen Avantgarden des 20. Jahrhunderts, auch wenn diese mit anderen medialen Konstellationen arbeiteten. Gerade im Hinblick auf deren Analyse hat sich für die Medienästhetik insbesondere der Begriff der Intermedialität als zentral erwiesen, und zwar aufgrund der ihm inhärenten Paradoxie: Denn um von Intermedialität als Vermischung medialer Strukturen sprechen zu können, benötigt man einen relativ eindeutigen Begriffvom Charakter und von den Funktionen der Medien, die jeweils intermedial zusammengefügt werden. Dies wird besonders deutlich in der Diskussion um die ästhetische Rolle der digitalen Medien: Ausstellungen wie Interact! (Wilhelm Lehmbruck Museum, Duisburg 1997), Schrift und Bild in Bewegung (Gasteig und Rathausgalerie, München 2000) oder poes1s. Digitale Poesie (Kulturforum Potsdamer Platz, Berlin 2004) heben Ende der 1990er/Anfang der 2000er Jahre insbesondere auf die materiellen Möglichkeiten ab, die nur die digitalen Medien bereithalten. ${ }^{2}$

Insofern bewegen sich die Diskurse um Medienkunst immer schon in einem Spannungsfeld zwischen medienspezifischen/-materiellen Eigenschaften und deren Verschwimmen, wenn nicht gar Verschwinden in intermedialen Konstellationen - eine Diagnose, die Janna Houwen 
zum Anlass für eine medientheoretische und medienästhetische Überprüfung nimmt. In ihrer 2017 erschienenen Monografie zum intermedialen Verhältnis von Film und Video steht die Frage nach der Medienspezifik schon im Untertitel. Houwen beginnt ihre Ausführungen zunächst mit einer Diskussion der Paradoxie des Intermedialitätsbegriffs: "Thus, models of intermediality which supposedly demonstrate the end of medium specificity, are often implicitly based on an originary ground on which media do have essences, are fixed, and achieve a final form." (S. 5) Diese essenzialisierende Perspektive kritisiert Houwen insofern, als sie - mit Rekurs auf Rosalind Krauss - dem technologischen Determinismus eine konventionalisierte Mediennutzung gegenüberstellt: Aus vier verschiedenen Blickwinkeln heraus diskutiert sie am Beispiel des Verhältnisses von Film und Video, inwieweit bestimmte Eigenschaften den jeweiligen Medien tatsächlich reingeschrieben s sind und wo soziale Zuschreibungen des Mediengebrauchs sich in einer gewissen Standardisierung der Funktionen niedergeschlagen haben, die ganz und gar nicht in der technischen Struktur des Mediums angelegt sind. Die vier Themenschwerpunkte umfassen den Realitätseffekt, das Phänomen des (dis)embodiment, soziale Strukturen sowie Aspekte der Gewalt.

In allen Kapiteln ist die Theoriediskussion eng mit konkreten künstlerischen Beispielen verflochten. So wird im Falle der Realitätseffekte besonders deutlich, wie Filmregisseur_innen die konventionalisierten Einsatzformen vor allem der Videotechnologie reproduzieren: In Michael Hanekes Film Benny's Video (A/CH 1992) übernehmen Videosequenzen dokumentarische Funktion und erzeugen mit ihrem Amateurcharakter (verwackelte Bilder, schlechte Farbqualität) Präsenz- und Unmittelbarkeitseffekte. Atom Egoyans Film Family Viewing (CA 1987) stellt das Videofilmen dem exzessiven Fernsehgenuss gegenüber, wobei Ersteres als Überwachungsaktivität in einen Gegensatz zur Passivität des Fernsehens gestellt wird. Krassimir Terzievs Dokumentation Battles of Troy ( $\mathrm{CH}$ 2005) über die fragwürdigen Bedingungen, unter denen die Massenszenen des Blockbusters Troja (Regie: Wolfgang Petersen, USA/MT/UK 2004) gedreht wurden, arbeitet schließlich mit von den Statist_innen während der Dreharbeiten heimlich aufgenommenen Videosequenzen, die als Authentizitätsgaranten einen wesentlichen Anteil an der Beweisführung der Dokumentation haben.

Zunächst scheint sich damit zu bestätigen, was Houwen eigentlich in Frage stellen will, nämlich eine

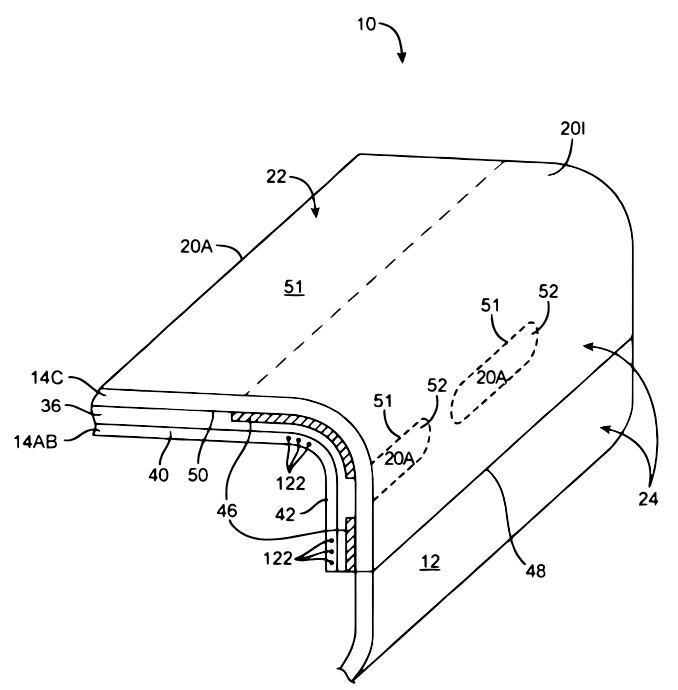

Inhärenz von medialen Eigenschaften, die beim Video in der Ad-hoc-Produktion und damit der Herstellung eines «referential-reality effect» liegen, beim Film in dessen hochgradiger Technizität und medienästhetischer Gestaltbarkeit, die einen "constructed-reality effect» erzeugt (siehe S. 51 ff.). Doch genau in dieser Zuspitzung wird der konventionalisierte Aspekt solcher Zuschreibungen manifest: «lt is through the typicality of certain features that the two media are specified and differentiated from each other. However, none of this relies on unique, inevitable technological features. It is mostly a conventional distinction.» (S. 58 )

In ähnlicher Weise, aber mit jeweils verschiedenen Rahmungen und Referenzen diskutiert Houwen auch die anderen Aspekte: Unter dem Titel «(Dis)embodiment» verhandelt sie auf der Basis von Jean Baudrys Theorie des Kino-Dispositivs und Laura Marks' Konzept des haptic cinema die Frage der medialen Konstellationen und ihrer Rezeptionsmodi. Dabei greift sie auf die dualistische Zuspitzung von Margaret Morse zurück, die zwischen proszenischen Künsten (Film) und Präsentationskünsten (Installation) unterscheidet. Erstere fesseln im Sinne von Baudry die Zuschauer_innen an ihre Sitze und konfrontieren sie mit der Leinwand, während Letztere - als Installationen - das Publikum integrieren, wodurch es gesamtleiblich wahrnehmen kann (vgl. S. 94). Auch hier wartet Houwen mit überzeugenden Beispielen auf, um diese Differenzierung nach und nach aufzulösen.

In den letzten beiden Teilen wird dann der rein ästhetische Raum der intermedialen Verhältnisse verlassen, 
um die gesellschaftlichen Dimensionen («Social Structures») sowie die Machtverhältnisse im Hinblick auf die Gewalt- und Diskriminierungspotenziale («Violent Features") beider Medien zu analysieren. Dabei scheint sich erneut ein standardisiertes Zuschreibungsverhältnis zu zeigen: Video eignet sich danach primär zum Individual- und Bekenntnismedium, während der Film das Zeug zum öffentlich-aktivistischen Protestmedium hat - eine Wertung, die eng mit den jeweiligen Produktionsbedingungen verbunden ist. Doch auch hier wird deutlich, dass diese Kennzeichnungen weniger medieninhärent als konventionalisiert sind - letztlich gibt es doch nur eine "ssoft determination" (Raymond Williams), gemäß der Medien als «work on a material for a specific purpose within necessary conditions'" definiert werden (Williams zit. n. Houwen, S. 157). Dies zumindest ist eine der wichtigsten Quintessenzen dieser durchgehend auf hohem Niveau argumentierenden Monografie.

Eine auf den ersten Blick ganz andere Herangehensweise an intermediale Kunst wählt der aus einem DFG-Forschungsprojekt hervorgegangene Band The Literariness of Media Art. Claudia Benthien, Jordis Lau und Maraike M. Marxsen legen eine breit angelegte Studie zur Medienkunst vor, die sich auf die Spurensuche nach der Arbeit mit Sprache in audiovisuellen Medien begibt. Es liegt allerdings angesichts der methodischen Zäsur durch die Postmoderne und die dadurch erfolgte Erweiterung des literaturwissenschaftlichen Diskurses auf kulturwissenschaftliche Fragen nicht unbedingt

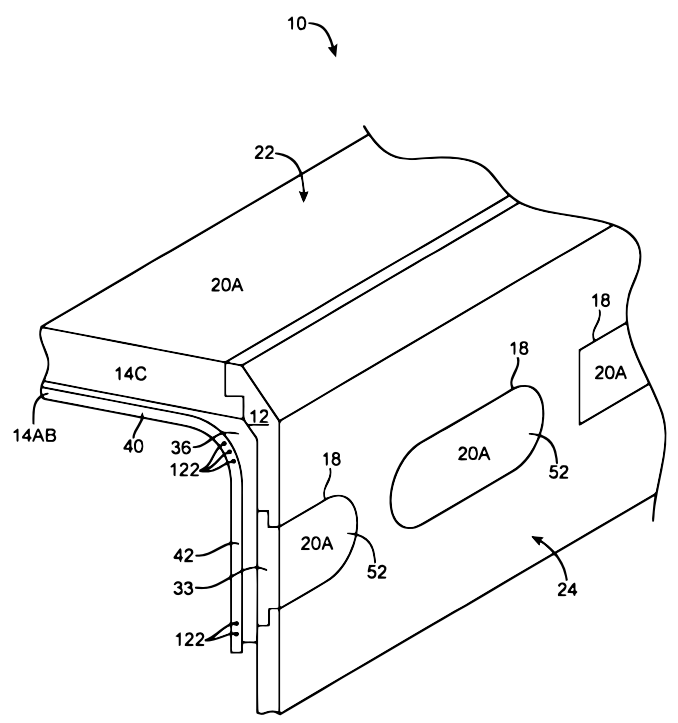

nahe, dies - wie es hier geschieht - mit Rekurs auf den Russischen Formalismus zu tun. Doch erweist sich diese Referenz als schlüssig begründet, und zwar durch die deutliche Traditionslinie der Medienkunst zu den frühen Avantgarden, deren ästhetische Experimente mit geschriebener und gesprochener Sprache - so die Autorinnen - die Grundlage für die prinzipiell transmediale Sprachauffassung der russischen Formalisten bildet (vgl. S. 7). Argumentativ schließt die Studie - trotz anderer Schwerpunktsetzung - sogar deutlich an Houwen an, da Benthien et al. ebenfalls die Kritik an der Konzentration der medienwissenschaftlichen Diskurse auf die Medienmaterialität zum Ausgangspunkt für die Suche nach einem erweiterten Blickwinkel machen.

Der Russische Formalismus ist - so die These - derart breit perspektiviert, dass er Analyseinstrumente für trans- und intermediale Phänomene bereitstellt, die als Scharnier zu aktuellen, zwischen Literatur- und Medienwissenschaft angelegten Diskursen fungieren können, beispielsweise zu Julia Kristevas Intertextualitätstheorie, zu Jay David Bolters und Richard Grusins RemediationKonzept und zu Ludwig Jägers Transkriptions-Modell oder zu den Filmwissenschaften (mit Rekurs u. a. aufTom Gunning, Vivian Sobchack und Laura Marks, vgl. S. 39 ff.). Wahrnehmung, Materialität und soziale Faktoren würden, so die Autorinnen, zudem im Russischen Formalismus gleichermaßen berücksichtigt (vgl. S. 21). Vor allem aber greifen sie die Befunde zur Ostranenie auf, also zur verfremdenden Wirkung von Literatur und Poesie, die deren ästhetischen Charakter ausmache - und zwar in ihren jeweiligen akustischen wie auch schriftlichen Manifestationen. Das ist es auch, was die Autorinnen mit «Literarizität) meinen. Da diese jedoch als transmediales Phänomen aufgefasst wird, variiert ihr Charakter von Medium zu Medium. Daher kann insbesondere die Medienkunst in ihrer grundlegenden intermedialen Anlage zwischen Visualität und Auditivität, die sowohl Text als auch gesprochene Sprache integriert, diese mit dem Bild und der Bewegung verbindet und so eine gesamtleibliche Rezeption herausfordert, als Neubelebung (und letztlich auch Erweiterung) der Sprachästhetik aufgefasst werden: «Media art contributes to a revitalization of language and awareness, and connects the linguistic and sensorial aspects of this experience.» (S.43)

Ausgehend von dieser theoretischen Rahmung untersuchen die Autorinnen eine Vielzahl von Film- und Videoarbeiten sowie Medienkunstinstallationen ${ }^{3}$ von 
den 196oer Jahren bis zur Gegenwart - zum einen im Hinblick auf die Integration von Text und Stimme, zum anderen in Bezug auf die Integration literarischer Genres (Poesie, Drama, Prosa), um abschließend ihr Augenmerk auf Adaptionen literarischer Werke in der Medienkunst zu richten. Im Gegensatz zu Houwen, die sich auf wenige Beispiele beschränkt (und damit das Risiko eingeht, zu selektiv vorzugehen), entscheiden sich Benthien et al. für eine überblicksartige Materialsammlung, die einen Zeitraum von nahezu Go Jahren umfasst und vor allem Beispiele aus dem deutschsprachigen und anglophonen Raum berücksichtigt. Diese Vielfalt hat Vor- und Nachteile: Die Vorteile liegen eindeutig in der geradezu kompendienhaften Übersicht, mit der Literarizität in den unterschiedlichsten audiovisuellen Formaten herausgearbeitet wird. Die Nachteile bestehen - und das verwundert angesichts des Mediums Buch nicht - in erster Linie darin, dass trotz zahlreicher Schwarzweiß-Abbildungen sehr viel Arbeit auf die Beschreibung der Werke verwendet werden muss und so die analytische Ebene teilweise in den Hintergrund rückt. Auch gerät durch die thematische Gruppierung im Hinblick auf die verschiedenen Formen von Literarizität die zeit- und kulturspezifische Einordnung der Werke teilweise aus dem Blick. Wenn beispielsweise in Bezug auf Peter Weibels partizipative Performance Das Recht mit Füßen treten von 1968 vornehmlich auf die Frage nach dem Verhältnis von Signifikat und Signifikant Bezug genommen wird, dann vermisst man die Referenz auf die Protestkunst im Kontext der 68er-Bewegung (vgl. S. 103 f.). Doch das ist Kritik auf hohem Niveau: Was hier auch von den beiden (noch nicht promovierten) Mitautorinnen Lau und Marxsen geleistet wird, ist beeindruckend in der theoretischen Reflexionstiefe wie auch in der Vielzahl der angeführten Beispiele.

Der Sammelband On the Fringes of Literature and Digital Media Culture von 2018 geht auf eine 2015 in Breslau abgehaltene Tagung zurück, die sich aus literatur-, filmund medienwissenschaftlicher Perspektive mit inter- und transmedialen Erzählformen beschäftigte. Als Anthologie naturgemäß heterogener als die beiden anderen Publikationen, spannt der Band ein breites Spektrum an Themen auf, die nicht nur an die Untersuchungen zu digitaler Literatur und ihrer verschiedenen Spielarten aus dem anglophonen Raum anschließen, sondern auch einen Blick auf ästhetische Produktionen in Osteuropa ermöglichen. Dabei werden im Unterschied zu den anderen beiden Bänden, die sich schwerpunktmäßig

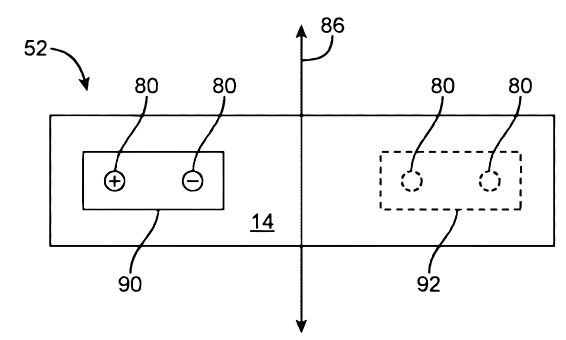

mit autor_innenzentrierten Kunstprojekten beschäftigen, vermehrt inter- und transmediale sowie vernetzte Schreib- und Rezeptionspraktiken in den Fokus gerückt. Agata Zarzycka beschäftigt sich beispielsweise mit den vielfältigen Aneignungen von Edgar Allan Poe und seinen Erzählungen im Netz (S.46-6o) und kann überzeugend herausarbeiten, dass sich in verschiedenen subkulturellen Szenen der Popkultur so etwas wie ein ikonischer Umgang mit der Person Poe konstituiert hat, der Mythenbildung mit Kommerz verbindet. Dabei verselbstständigt sich die Appropriation der Person Poes und seiner Geschichten zu eigenständigen ästhetischen Konstrukten, wie beispielsweise in den real-person fictions, die Person und Motive seiner Erzählungen miteinander verquicken (vgl. S. 54).

Aleksandra Małecka und Piotr Marecki beschäftigen sich mit einer anderen Form digitaler Textproduktion, nämlich der Übersetzung literarischer Werke mittels algorithmischer Programme - in diesem speziellen Fall geht es um die Urfassung des Theaterstücks Ubu Roi, das 1888 als Marionettentheater unter dem Titel Ubu Roi ou les Polonais von Alfred Jarry, damals noch Schüler, verfasst wurde: Die Autor_innen haben dieses Werk mittels Google Translate ins Polnische übersetzt und das Ergebnis in Buchform veröffentlicht. Die Grundfrage, die hier verhandelt wird, ist nicht etwa die nach der sprachlichen und semantischen Angemessenheit der Übersetzung, sondern nach den praxeologischen Implikationen eines solchen Vorgehens. Dabei rückt vor allem die Frage nach der Kreativität in den Fokus, die zunächst mit Referenz auf Kenneth Goldsmith beantwortet wird:

Thus, we propose to interpret the GT [Google Translate] translation [...] as an «uncreative translation» mirroring the term «uncreative writing" introduced by Goldsmith. In this light, appropriating even an entire text in a way that is meaningful and innovative can legitimately be considered artistic expression and a new work. (S.81) 
Bei näherer Betrachtung der Komplexität der Datenbank, die Google Translate zugrunde liegt, enthüllt sich nämlich ein eminent kreativer Charakter der (scheinbar) automatisierten Übersetzung: Für diese greift der Algorithmus nicht nur auf ältere, nichtmaschinell entstandene Übersetzungen des Werks zurück, sondern stellt auch intertextuelle Bezüge zu ähnlichen Topoi der polnischen Literatur her, die monströse und dehumanisierte Gestalten (wie Ubu Roi eine ist) zum Thema haben (vgl. S. 84). Auf diese Weise wird eine datenbankbasierte Intertextualität zur Basis für die automatisierte Übersetzung, die dann doch wieder auf menschliche Leistung (nämlich andere literarische Werke) verweist.

Der gesamte Band erweist sich als spannendes Mosaik verschiedenster Schreibpraktiken, die nicht immer digital sind. Dirk de Geest beispielsweise analysiert Handbücher kreativen Schreibens als aussagekräftige Dokumente für die Analyse literarischer Dynamiken, in denen sich - wie in Kreativitätsratgebern generell - das Spannungsfeld zwischen Regelhaftigkeit und arkanischem Genius oder, etwas bodenständiger ausgedrückt, "this tension between inspiration and transpiration" manifestiert (S. 89-102, hier S. 93). Weitere Beiträge zu Narrativität in Computerspielen, zum transmedialen Erzählen, zur Blogosphäre in Polen sowie abschließend zum product placement in literarischen Erzählungen runden den sehr empfehlenswerten Band zur Vielfalt von ästhetischen Schreibpraktiken in unterschiedlichen medialen Konstellationen ab.

So divergent die drei Publikationen in ihren Herangehensweisen auch auftreten, sie zeigen doch eines sehr deutlich: Die Bezüge der Medienkunst, sei sie primär bild- oder textbasiert oder genuin intermedial verfasst, sind derart komplex und vielfältig, dass sie unterschiedliche Herangehensweisen und theoretische wie ästhetische Kontextualisierungen rechtfertigen, ja sogar erfordern. Aber genau darin sind auch wieder Gemeinsamkeiten und perspektivische Komplementaritäten zu entdecken: sei es in den Versuchen, den Begriff der Intermedialität besser zu fassen, sei es in der Erkenntnis, dass ästhetische Produktion auch auf ihre sozialen Kontexte verweist - oder in der immer wieder neu zu stellenden Frage, was uns Medienkunst nun eigentlich über uns selber und unsere Zeit mitteilen kann.

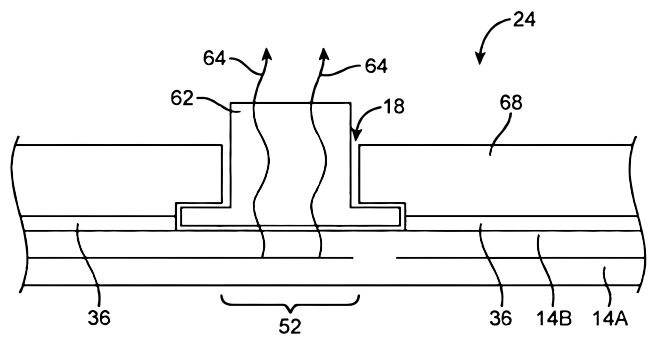

1 Zu nennen wären im deutschsprachigen Bereich z. B. Söke Dinkla: Pioniere interaktiver Kunst von 1970 bis heute. Myron Krueger, Jeffrey Shaw, David Rokeby, Lynn Hershman, Grahame Weinbren, Ken Feingold, Ostfildern 1997; Kai-Uwe Hemken: Bilder in Bewegung. Traditionen digitaler Ästhetik, Köln 2000; Peter Gendolla u. a. (Hg.): Formen interaktiver Medienkunst, Frankfurt/M. 2001. Die letztgenannte Publikation erschien mit einer Mini-CD-ROM.

2 Zu dieser Zeit war Kunst mit digitalen Medien noch eine Marginalie im Kunstbetrieb. Die genannten Ausstellungen trugen wesentlich dazu bei, sie einer größeren Öffentlichkeit zugänglich zu machen und mussten daher auf ihre Spezifika abheben. Zu den Ausstellungen vgl. die entsprechenden Kataloge bzw. Begleitpublikationen: Söke Dinkla (Hg.): Interact! Schlüsselwerke interaktiver Kunst, Ostfildern 1997; Bernd Scheffer (Hg.): Schrift und Bild in Bewegung, München 2000; Friedrich W. Block, Christiane Heibach, Karin Wenz (Hg.): poes1s. Digitale Poesie/Digital Poetry, Ostfildern 2004.

3 (Medienkunst) wird hier in Abgrenzung von Film- oder Videoarbeiten, die nicht installativ auftreten, verwendet und bezeichnet alle Installationen, die mit Film, Video und/oder digitalen Medien arbeiten. 